\title{
Circ_0056285 Regulates Proliferation, Apoptosis and Glycolysis of Osteosarcoma Cells via miR-I244/TRIM44 Axis
}

This article was published in the following Dove Press journal: Cancer Management and Research

\author{
Shousong Huo' \\ Dongmei Dou ${ }^{2}$ \\ 'Department of Orthopaedic, Huaihe \\ Hospital of Henan University, Kaifeng, \\ 475000, Henan, People's Republic of \\ China; ${ }^{2}$ Institute of Chronic Disease Risk \\ Assessment, Henan University, Jinming \\ Campus, Kaifeng, 475000, Henan, \\ People's Republic of China
}

Background: Osteosarcoma (OS) is a common malignant bone cancer that occurs in adolescents and children. Circular RNAs (circRNAs) are important regulators of tumorigenesis and development. This study aimed to explore the role and molecular basis of circ_0056285 in OS.

Methods: The levels of circ_0056285, miR-1244 and tripartite motif containing 44 (TRIM44) were determined by quantitative real-time polymerase chain reaction or Western blot assay. Cell proliferation was evaluated by Cell Counting Kit-8 (CCK-8) assay and colony formation assay. Cell apoptosis was assessed by flow cytometry and caspase 3and caspase 9 activity assay kits. Glucose uptake, lactate product and ATP level were examined using commercial kits. Hexokinase II (HK2) and lactate dehydrogenase A (LDHA) levels were measured by Western blot assay. The interaction among circ_0056285, miR-1244 and TRIM44 was confirmed by dual-luciferase reporter assay, RNA immunoprecipitation assay or RNA pull-down assay. Xenograft experiment was conducted to explore tumor growth in vivo. Exosomes were identified by transmission electron microscope (TEM), nanoparticle tracking analysis (NTA) and Western blot. The diagnostic value of exosomal circ_0056285 was evaluated by receiver operating characteristic (ROC) curve.

Results: Circ_0056285 and TRIM44 were up-regulated, and miR-1244 was down-regulated in OS tissues and cells. Circ_0056285 silencing inhibited proliferation and glycolysis and promoted apoptosis in OS cells. Also, circ_0056285 knockdown hindered proliferation and accelerated apoptosis in OS cells by regulating miR-1244/TRIM44 axis. Circ_0056285 depletion impeded tumor growth in vivo. Furthermore, ROC curve showed that circ_0056285 might be a diagnostic biomarker in OS.

Conclusion: Circ_0056285 facilitated OS progression by sponging miR-1244 and increasing TRIM44 expression, providing a promising therapeutic target for OS.

Keywords: osteosarcoma, circ_0056285, miR-1244, TRIM44, progression
Correspondence: Dongmei Dou Institute of Chronic Disease Risk Assessment, Henan University, Jinming Campus, Kaifeng, 475000, Henan,

People's Republic of China

Tel +86-037I-23885066

Email doudongmei@henu.edu.cn

\section{Introduction}

Osteosarcoma (OS) is a common malignant bone cancer with the highest incidence in childhood and adolescence. ${ }^{1}$ With the improvement of combination treatment, the 5-year survival rate of OS patients has reached $70 \%{ }^{2}$ Nevertheless, tumor metastasis and recurrence make the treatment of osteosarcoma more difficult. Hence, exploiting better treatment strategies is essential to improve the prognosis of OS.

Circular RNAs (circRNAs) are a new type of endogenous non-coding RNAs with covalent closed-loop structures. ${ }^{3}$ Increasing evidence manifested that 
circRNAs could function as miRNA molecular sponges to participate in gene regulation. ${ }^{4}$ Besides, some studies indicated that circRNAs exerted a crucial effect on biological function and pathogenic mechanism of osteosarcoma. ${ }^{5}$ For example, circ_001621 contributed to tumor growth and metastasis in OS via binding to miR-578 and activating VEGF. ${ }^{6}$ Circ-0001785 accelerated OS progression via regulating miR-1200/HOXB2 axis. $^{7}$ In addition, circ_0102049 aggravated the malignancy of osteosarcoma by down-regulating miR-1304-5p and elevating MDM2. ${ }^{8}$ However, the role of circ_0056285 in OS remains unknown. Previous research revealed that circ_0056285 was remarkably up-regulated in intracranial aneurysms. ${ }^{9}$ High-throughput sequencing results showed that circ_0056285 was highly expressed in osteosarcoma, ${ }^{10}$ and we speculated that circ_0056285 might be an oncogenic factor for osteosarcoma.

Moreover, a large number of miRNAs play a vital role in the occurrence and progression of osteosarcoma. ${ }^{11}$ For instance, inhibition of miRNA-181a impeded OS cell proliferation and metastasis by mediating NLRP3. ${ }^{12}$ Wang et al discovered that miR-320a up-regulation expedited OS progression via repressing CPEB1. ${ }^{13}$ Also, miR-33b targeted LDHA to suppress OS cell growth and glycolysis. ${ }^{14}$ In addition, previous research unveiled that miR-1244 was prominently down-regulated in OS. ${ }^{15}$ However, the relationship between circ_0056285 and miR-1244 has not been studied.

Tripartite motif containing 44 (TRIM44) belongs to TRIM family and lacks RING domain. ${ }^{16}$ Numerous studies have evidenced that TRIM44 is a promoter of many cancers. In renal cell carcinoma, TRIM44 accelerated tumor development by down-regulating FRK. ${ }^{17}$ In colorectal cancer, TRIM44 expedited cell growth and metastasis via triggering Akt/mTOR pathway. ${ }^{18}$ Also, TRIM44 was a crucial prognostic marker for malignant tumors. ${ }^{19,20}$

Therefore, we first detected the expression of circ_0056285 in OS tissues, cells and serum exosomes. Furthermore, we investigated the function of circ_0056285 in OS and its potential molecular mechanism.

\section{Materials and Methods}

\section{Serum and Tissue Samples}

Thirty-five pairs of OS patients and healthy volunteers were recruited from Huaihe Hospital of Henan University. Serum samples were obtained by centrifugation of $5 \mathrm{~mL}$ venous blood provided by each participant. Thirty-five OS tissues and matched adjacent normal tissues were collected from OS patients. This research was ratified by the Ethics Committee of Huaihe Hospital of Henan University and was carried out according to the guidelines of Declaration of Helsinki. All participants signed written informed consent.

\section{Cell Culture}

Human osteoblast cells (hFOB1.19) and OS cell lines (143B, MG63, U2OS and HOS) were purchased from American Type Culture Collection (ATCC, Manassas, VA, USA). All cells were cultured in Dulbecco's Modified Eagle Medium (DMEM; Gibco, Carlsbad, CA, USA) supplemented with $10 \%$ fetal bovine serum (FBS; Gibco) at $37^{\circ} \mathrm{C}$ with $5 \% \mathrm{CO}_{2}$.

\section{Cell Transfection}

Small interfering RNA (siRNA) against circ_0056285 (sicirc\#1 and si-circ\#2), siRNA control (si-NC), miR-1244 mimics (miR-1244), the mimics control (miR-NC), circ_0056285 overexpression vector (oe-circ_0056285), TRIM44 overexpression vector (oe-TRIM44), the empty vector (vector), miR-1244 inhibitor (anti-miR-1244) and the inhibitor control (anti-NC) were obtained from Ribobio (Guangzhou, China). Lipofectamine 3000 (Invitrogen, Carlsbad, CA, USA) was used to transfect oligonucleotides and plasmids into 143B and HOS cells.

\section{Quantitative Real-Time Polymerase Chain Reaction (qRT-PCR)}

Total RNA was extracted using Trizol reagent (Invitrogen). Then, RNA was reverse-transcribed using HiScript II One Step RT-PCR Kit (Vazyme, Nanjing, China) or miScript II RT Kit (Qiagen, Frankfurt, Germany). The expression level was detected using AceQ qPCR SYBR Green Master Mix (Vazyme) and calculated using $2^{-\Delta \Delta \mathrm{Ct}}$ method. The primers were as follows: circ_0056285-F: 5'-TGACAAATCGGTGGAGG ACG-3', circ_0056285-R: 5'-CACTGAAGAGCAGGGA CCAG-3'; miR-1244-F: 5'-TTACAAAGTAGTTGGTTTG TATGAG-3', miR-1244-R: 5'-TGGTGTCGTGGAGTCG -3'; TRIM44-F: 5'-GTGGACATCCAAGAGGCAAT-3', TRIM44-R: 5'-AGCAAGCCTTCATGTGTCCT-3'; GAP DH-F: 5'-GGGAAACTGTGGCGTGAT-3', GAPDH-R: 5'-GAGTGGGTGTCGCTGTTGA-3'; U6-F: 5'-CTCGCT TCGGCAGCACA-3', U6-R: 5'-AACGCTTCACGAAT TTGCGT-3'. GAPDH or U6 was used as internal control. 


\section{Actinomycin $\mathrm{D}$ and RNase $\mathrm{R}$ Treatment}

To restrict transcription, $2 \mathrm{mg} / \mathrm{mL}$ Actinomycin D (Seebio, Shanghai, China) was added to OS cell medium. For RNase R treatment, $2 \mu \mathrm{g}$ RNA was incubated with or

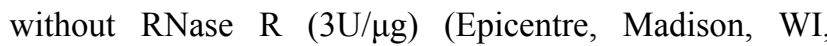
USA) for $30 \mathrm{~min}$ at $37^{\circ} \mathrm{C}$.

\section{RNA Isolation from Nuclear and Cytoplasmic Fractions}

The nuclear and cytoplasmic fractions of OS cells were isolated using the PARIS kit (Invitrogen). 18S rRNA and U6 were employed as positive controls for cytoplasmic and nuclear fractions, respectively.

\section{Cell Counting Kit-8 (CCK-8) Assay}

Cells $\left(3.0 \times 10^{3}\right)$ were placed into 96 -well plates. After culture $0 \mathrm{~h}, 24 \mathrm{~h}, 48 \mathrm{~h}$ and $72 \mathrm{~h}, 10 \mu \mathrm{L}$ CCK-8 solution (Solarbio, Beijing, China) was added. After incubation for another $2 \mathrm{~h}$, the optical density was measured at $450 \mathrm{~nm}$ using a Microplate Reader (BioTek, Burlington, VT, USA).

\section{Colony Formation Assay}

OS cells were seeded into six-well plates and cultured at $37^{\circ} \mathrm{C}$ for 14 days in a medium containing $10 \%$ FBS. Next, after fixing with methanol and staining with $0.5 \%$ crystal violet, the number of colonies was imaged and counted.

\section{Cell Apoptosis Assay}

Cells were plated into six-well plates and digested with trypsin. After washing with PBS for three times, AnnexinV-fluorescein isothiocyanate (AnnexinV-FITC) and Propidium Iodide (PI) were added and incubated for $15 \mathrm{~min}$. Then, the apoptotic rate was detected using an Attune NxT Flow Cytometer (Thermo Fisher Scientific, Waltham, MA, USA). In addition, the activities of caspase 3 and caspase 9 were examined using caspase 3 and caspase 9 activity assay kits (Beyotime, Shanghai, China) according to the manufacturer's instructions.

\section{Measurement of Glucose Uptake, Lactate Product and ATP Level}

Glucose uptake and lactate product were detected by Glucose and Lactate Assay Kits (Sigma-Aldrich, St Louis, MO, USA) following the manufacturer's requirements. ATP level was measured using ATP colorimetric assay kit (Abcam, Cambridge, UK) according to the manufacturer's instructions.

\section{Western Blot Assay}

Cells were lysed with RIPA buffer (Solarbio). Subsequently, proteins were separated by polyacrylamide gel electrophoresis and transferred to polyvinylidene fluoride (PVDF) membranes (Millipore, Billerica, MA, USA). After blocking with 5\% skim milk for $1 \mathrm{~h}$, the membranes were incubated with primary antibodies against hexokinase II (HK2; 1:5000, ab227198, Abcam), lactate dehydrogenase A (LDHA; 1:1000, ab125683, Abcam), TRIM44 (1:2000, ab236422, Abcam) or $\beta$-actin (1:2000, ab8227, Abcam). Next, the membranes were washed with TBST buffer 4 times. Then, the membranes were probed with secondary antibody (1:20000, ab205718, Abcam) for $2 \mathrm{~h}$ at room temperature and then washed with TBST buffer 4 times. All antibodies were diluted with TBST buffer. The protein bands were visualized using the enhanced chemiluminescence system (Qiagen).

\section{Dual-Luciferase Reporter Assay}

Circ_0056285 or TRIM44 3'UTR containing miR-1244 wild-type or mutant binding sites was cloned into pmirGLO vector (Promega, Madison, WI, USA) and named as WT-circ_0056285, MUT-circ_0056285, WTTRIM44 or MUT-TRIM44. The corresponding luciferase reporter and miR-1244 or miR-NC were co-transfected into 143B and HOS cells. The luciferase activity was examined by Dual-Lucy Assay Kit (Solarbio).

\section{RNA Immunoprecipitation (RIP) Assay}

EZ-Magna RIP kit (Millipore) was employed for RIP analysis. After lysing 143B and HOS cells with RIP lysis buffer, the cell lysates were incubated with magnetic beads bound with Ago2 or IgG antibodies. The enrichment of circ_0056285 and miR-1244 was measured by qRT-PCR.

\section{RNA Pull-Down Assay}

Biotin-labeled miR-1244 (bio-miR-1244) and the control (bio-miR-NC) were bought from Ribobio. 143B and HOS cells were lysed and then incubated with M-280 Streptavidin Dynabeads (Invitrogen). Then, circ_0056285 expression was examined using qRT-PCR.

\section{Xenograft Assay}

The animal experiment was approved by the Animal Ethics Committee of Huaihe Hospital of Henan University and performed in accordance with the 
guidelines of the National Animal Care and Ethics Institution. All animal procedures were conducted at the Animal Institute of Henan University. 143B cells were transfected with lentivirus containing circ_0056285 short hairpin RNA (sh-circ) or the control (sh-NC) (GenePharma, Shanghai, China). The stable transfected $143 \mathrm{~B}$ cells $\left(5 \times 10^{6}\right)$ were subcutaneously injected into the right-back of $\mathrm{BALB} / \mathrm{c}$ nude mice. The tumor size was measured using a digital caliper at indicated time points. After 35 days, the mice were sacrificed by cervical dislocation. If no spontaneous breathing was observed within 2-3 min and no blink reflex, the mice were considered euthanized. Then, the tumors were excised and weighed.

\section{Exosome Isolation and Identification}

Briefly, the serum was centrifuged at $3000 \times \mathrm{g}$ for $10 \mathrm{~min}$ to precipitate cells and then centrifuged at $10000 \times \mathrm{g}$ for $30 \mathrm{~min}$ to remove cell debris. Subsequently, the collected supernatant was ultracentrifuged at $110000 \times \mathrm{g}$ for $60 \mathrm{~min}$, and the exosomes were resuspended in PBS, followed by ultracentrifugation for $60 \mathrm{~min}$ to obtain pure exosomes. Then, the morphology and size of exosomes were observed using a Philips CM120 transmission electron microscope (TEM) (Philips Research, Eindhoven, Netherlands) at $80 \mathrm{kV}$. The size distribution of exosomes was analyzed by nanoparticle tracking analysis (NTA). The presence of exosome marker (CD61, CD81 and TSG101) was determined by Western blot assay.

\section{Statistical Analysis}

Graphpad Prism 7.0 software (GraphPad, San Diego, CA, USA) was utilized to analyze data. Data were shown as mean \pm standard deviation in three independent replicates. Differences were assessed using Student's $t$-test or oneway analysis of variance. $P<0.05$ was considered statistically significant.

\section{Results}

\section{Expression and Validation of Circ_0056285 in OS Cells}

Firstly, qRT-PCR analysis exhibited that circ_0056285 was significantly up-regulated in OS cell lines (143B, MG63, U2OS and HOS) compared with normal osteoblast cell line hFOB1.19 $(* * P=0.002$ or $* * * P<0.001$, Figure 1A). Then, Sanger sequencing was performed to verify head-to-tail splicing, and the results showed that circ_0056285 was derived from exons 2, 3 and 4 of
RALB gene (Figure 1B). In order to rule out the possibility of genomic rearrangement or trans-splicing, we designed divergent primers to amplify circ_0056285, and the results illustrated that circ_0056285 was amplified in cDNA, but not in gDNA (Figure 1C). Next, actinomycin $\mathrm{D}$ analysis showed that the half-life of circ_0056285 transcript exceeded $24 \mathrm{~h}$, suggesting that circ_0056285 was stable in 143B and HOS cells $(* * * P<$ 0.001, Figure 1D). Similarly, RNase R digestion assay revealed that circ_0056285 was resistant to RNase $\mathrm{R}(* * * P<0.001$, Figure 1E). Additionally, the subcellular localization of circ_0056285 was detected using nuclear and cytoplasmic fractions, and the results indicated that circ_0056285 was mainly distributed in the cytoplasm (Figure 1F). Taken together, these data suggested that circ_0056285 was dysregulated and stable in OS.

\section{Circ_0056285 Knockdown Inhibited Proliferation and Promoted Apoptosis in OS Cells}

To explore the role of circ_0056285 in OS cells, 143B and HOS cells were transfected with si-NC, si-circ\#1 or sicirc\#2. The knockdown efficiency of circ_0056285 was determined by qRT-PCR analysis $(* * * P<0.001$, Figure 2A). CCK-8 assay and colony formation assay exhibited that down-regulation of circ_0056285 decreased the proliferation ability of OS cells $(* * * P<0.001$, Figure $2 \mathrm{~B}$ and C). Also, flow cytometry showed that circ_0056285 silencing increased the apoptosis rate of 143B and HOS cells (***P<0.001, Figure 2D). Simultaneously, circ_0056285 knockdown elevated the activities of caspase 3 and caspase 9 in OS cells $(* * * P<0.001$, Figure $2 \mathrm{E}$ and $\mathrm{F})$. Overall, these data indicated that depletion of circ_0056285 inhibited proliferation and promoted apoptosis in OS cells.

\section{Circ_0056285 Silencing Suppressed the Glycolysis of OS Cells}

To investigate the effect of circ_0056285 on glycolysis in OS cells, RNA interference was used to silence circ_0056285 expression in 143B and HOS cells. As displayed in Figure 3A and B, silencing of circ_0056285 reduced glucose uptake and lactate product in 143B and HOS cells $(* * P=0.002$ or $* * * P<0.001)$. In addition, transfection with si-circ\#1 or si-circ\#2 led to a significant reduction in ATP level $\left(* * P=0.001,{ }^{* *} P=0.006,{ }^{* * *} P<\right.$ 
A

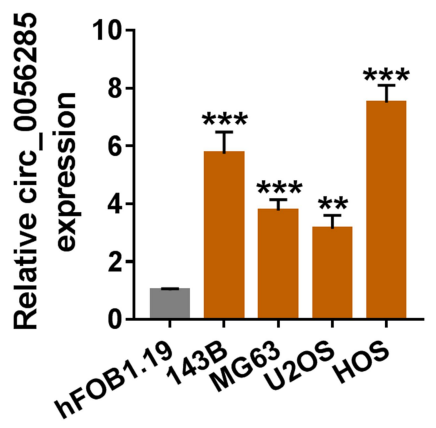

B

RALB Genomic DNA chr2:121036193-121047333[+]

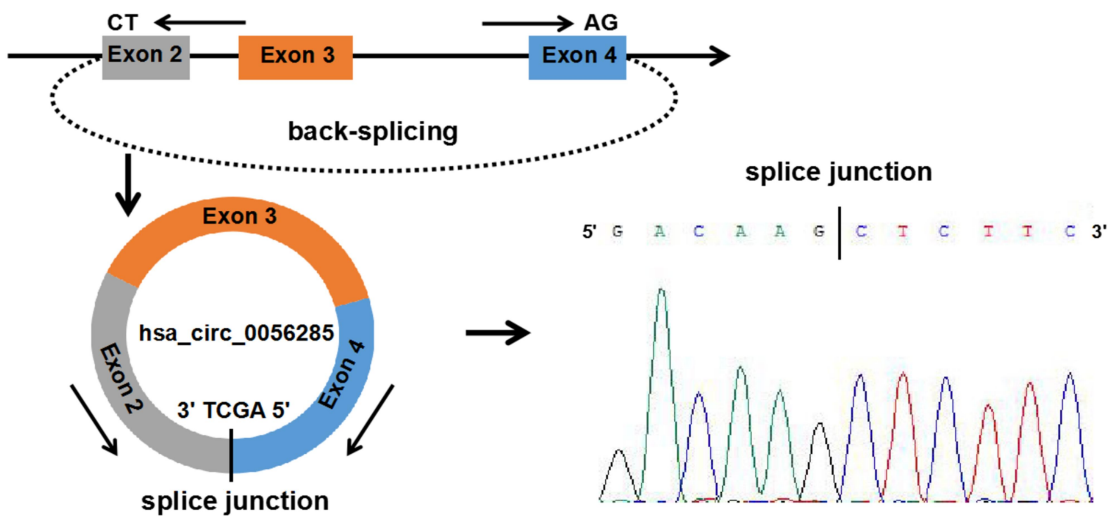

\section{C $143 B$}

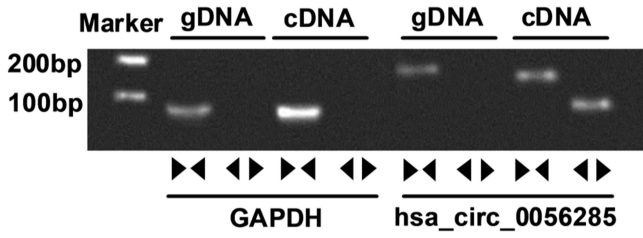

HOS

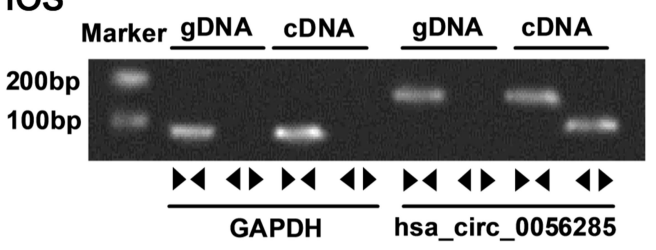

D
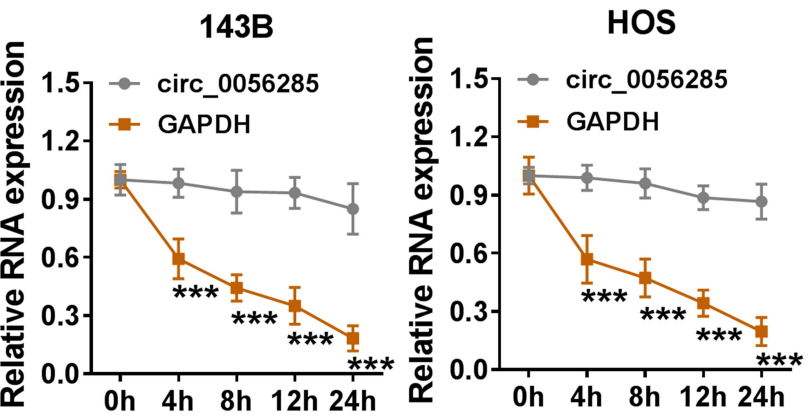

E
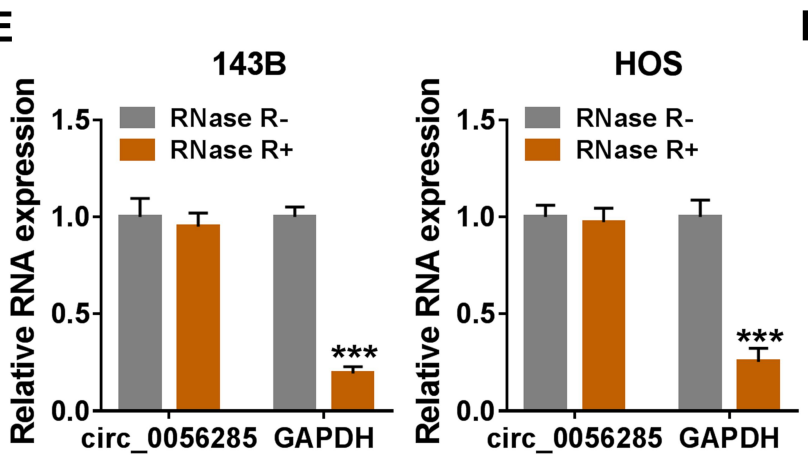

F
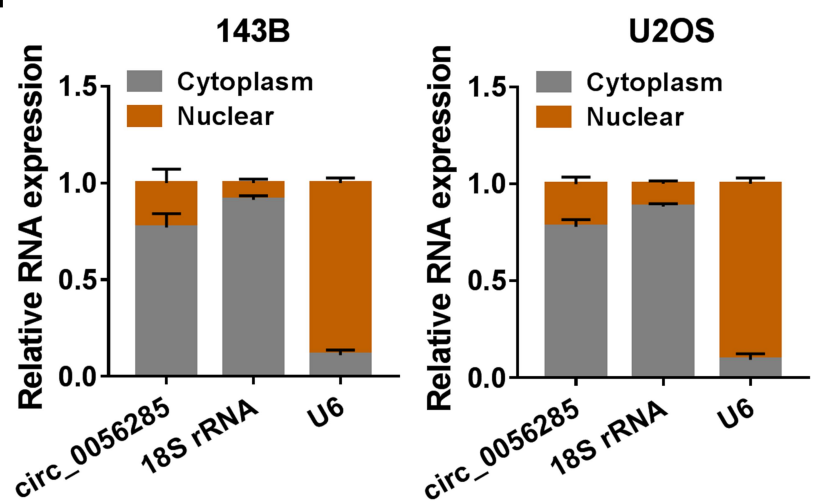

Figure I Expression and validation of circ_0056285 in OS cells. (A) The expression of circ_0056285 in hFOBI.19 and OS cell lines (I43B, MG63, U2OS and HOS) was measured by qRT-PCR. (B) The schematic illustration showed the origin of circ_0056285 and the result of Sanger sequencing. (C) Circ_0056285 was amplified by divergent primers in cDNA but not gDNA. (D) I43B and HOS cells were treated with Actinomycin D, and the expression of circ 0056285 and GAPDH was examined by qRT-PCR at different times. (E) The levels of circ_0056285 and GAPDH were detected in I43B and HOS cells treated with or without RNase R. (F) The levels of I8S rRNA, U6 and circ_0056285 in nuclear and cytoplasmic fractions were evaluated by qRT-PCR. **P $<0.01$, *** $2<0.001$.

0.001 , or $* * P=0.001$ Figure $3 \mathrm{C})$. Moreover, Western blot analysis revealed that knockdown of circ_0056285 resulted in a marked decrease in HK2 and LDHA levels $(* * * P<0.001$, Figure $3 \mathrm{D}$ and $\mathrm{E})$. These data indicated that circ_0056285 depletion inhibited the glycolysis of OS cells.

\section{Circ_0056285 Directly Interacted with miR-I 244}

To further explore whether circ_0056285 bound to miRNAs, circbank and CircInteractome predicted four potential target miRNAs (miR-1244, miR-548b-3p, miR-604 and miR-614) 

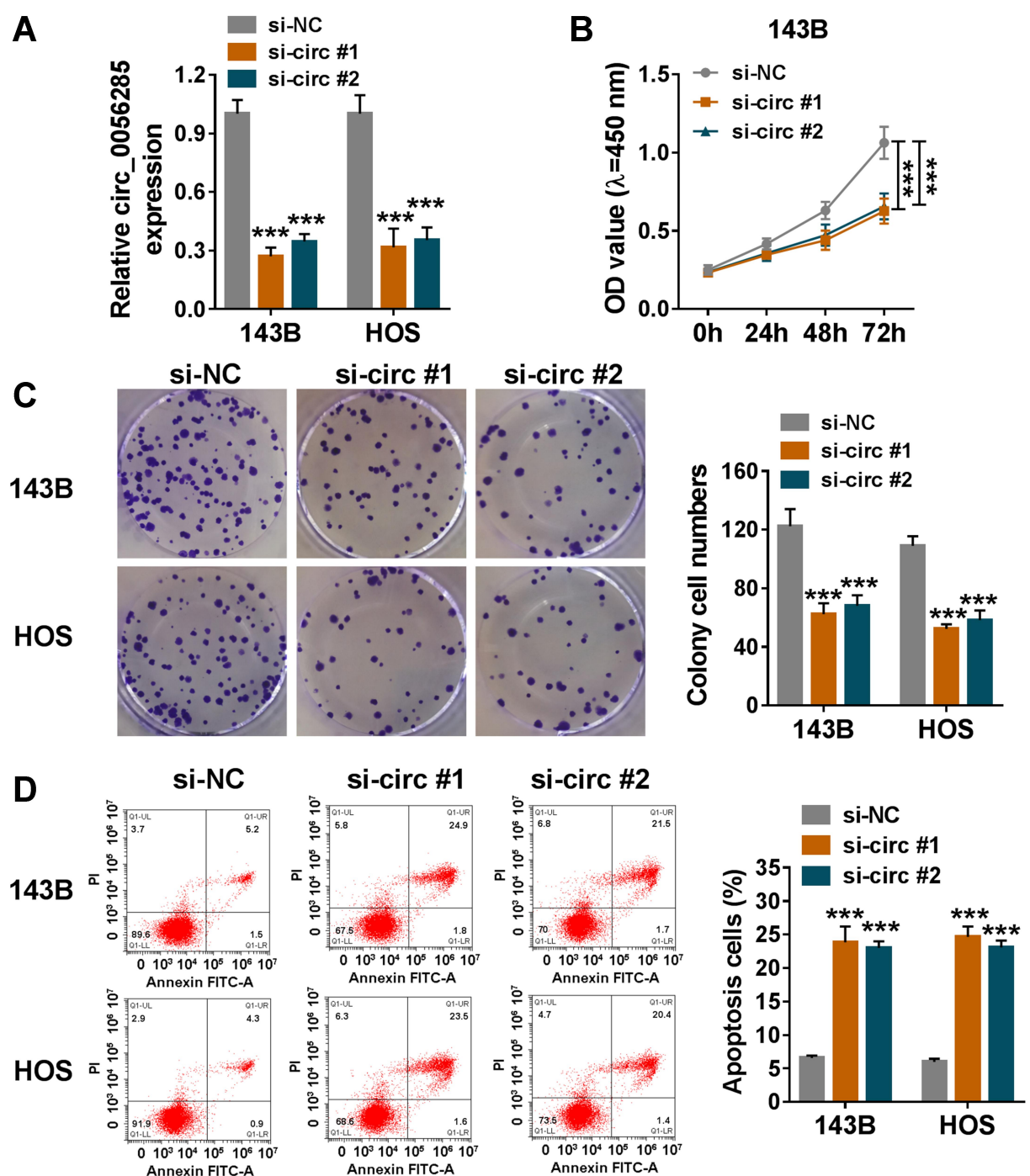

Figure 2 Circ_0056285 knockdown inhibited proliferation and promoted apoptosis in OS cells. I43B and HOS cells were introduced with si-NC, si-circ\#I or si-circ\#2. (A) The knockdown efficiency was assessed by qRT-PCR. (B and C) Cell proliferative capacity was evaluated by CCK-8 assay and colony formation assay. (D) Cell apoptosis was detected by flow cytometry. (E and $\mathbf{F})$ The activities of caspase 3 and caspase 9 were examined using commercial kits. $* * * p<0.001$.

for circ_0056285 (Figure 4A). qRT-PCR analysis showed that miR-1244 was most significantly up-regulated in 143B and HOS cells transfected with si-circ\#1 compared to the other three miRNAs $\left(* * * P<0.001,{ }^{* *} P=0.003\right.$, or $* * P=$ 0.004 , Figure 4B). In addition, miR-1244 expression was remarkably decreased in OS cells (143B, MG63, U2OS and HOS) compared with hFOB1.19 cells $(* * * P<0.001$, Figure $4 C)$. Next, 143B and HOS cells were introduced with miR1244 or miR-NC, and the overexpression efficiency of miR1244 was validated by qRT-PCR $(* * * P<0.001$, Figure 4D). The putative binding sites of circ_0056285 and miR-1244 are shown in Figure 4E. Dual-luciferase reporter assay exhibited that miR-1244 mimics strikingly reduced the luciferase activity of WT-circ_0056285 reporter $(* * * P<0.001$, Figure
4F). Simultaneously, RIP assay and RNA pull-down assay were performed to verify whether circ_0056285 directly targeted miR-1244. The results suggested that circ_0056285 and miR-1244 were markedly enriched in Ago2 group relative to $\operatorname{lgG}$ group $(* * * P<0.001$, Figure $4 \mathrm{G})$. Also, circ_0056285 was pulled down by bio-miR $-1244(* * * P<0.001$, Figure $4 \mathrm{H})$. Collectively, these data indicated that circ_0056285 was a sponge of miR-1244 in OS cells.

\section{Circ_0056285 Sponged miR-I 244 to Regulate TRIM44 Expression}

According to the prediction results of TargetScan database and relevant references, we initially selected three 
A

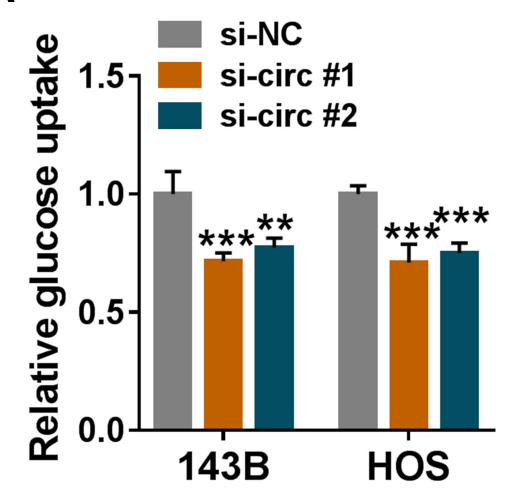

D

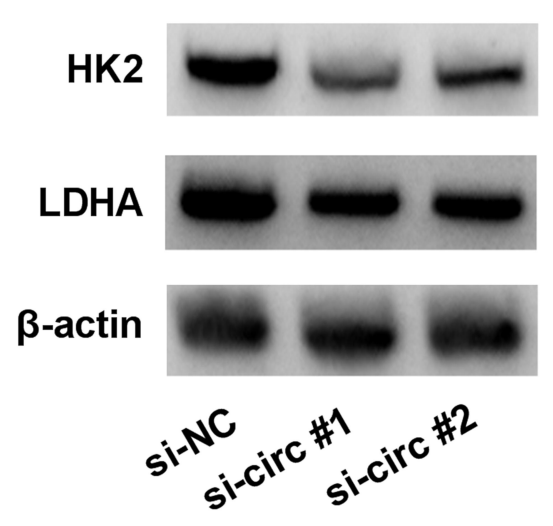

$\mathbf{E}$

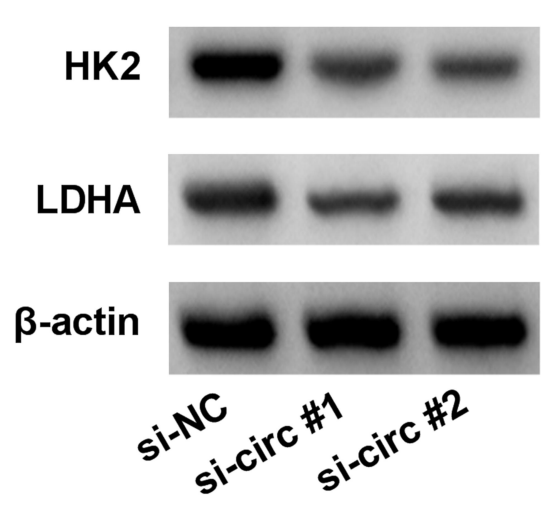

B

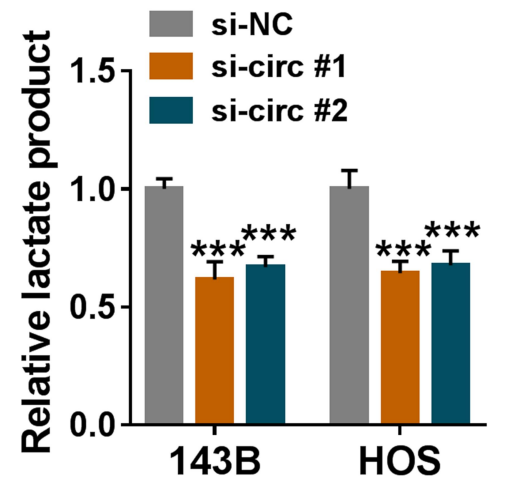

C

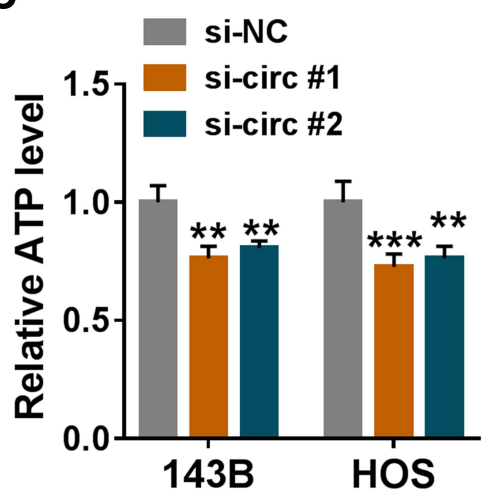

Figure 3 Circ_0056285 silencing suppressed the glycolysis of OS cells. I43B and HOS cells were transfected with si-NC, si-circ\#I or si-circ\#2. (A and B) After transfection for $48 \mathrm{~h}$, glucose uptake and lactate product were detected. (C) ATP level was measured using ATP colorimetric assay kit. (D and E) The levels of glycolysis-related proteins $(\mathrm{HK} 2$ and LDHA) were examined by Western blot assay. $* * P<0.01$, $* * * P<0.001$.

genes (TRIM44, TRIM2 and TRIM24) for research (Figures 5A and $\underline{\mathrm{S} 1 \mathrm{~A}}$ ). Then, TRIM44 was selected for subsequent experiments based on the results of dualluciferase reporter assay $(* * * P<0.001$, Figure S1B). The results revealed that mature miR-1244 significantly decreased the luciferase activity of WT-TRIM44 reporter $(* * * P<0.001$, Figure 5B). Compared with hFOB1.19 cells, the protein expression of TRIM44 was notably increased in 143B and HOS cells $(* * * P<0.001$, Figure 5C). Additionally, circ_0056285 had significant 
A
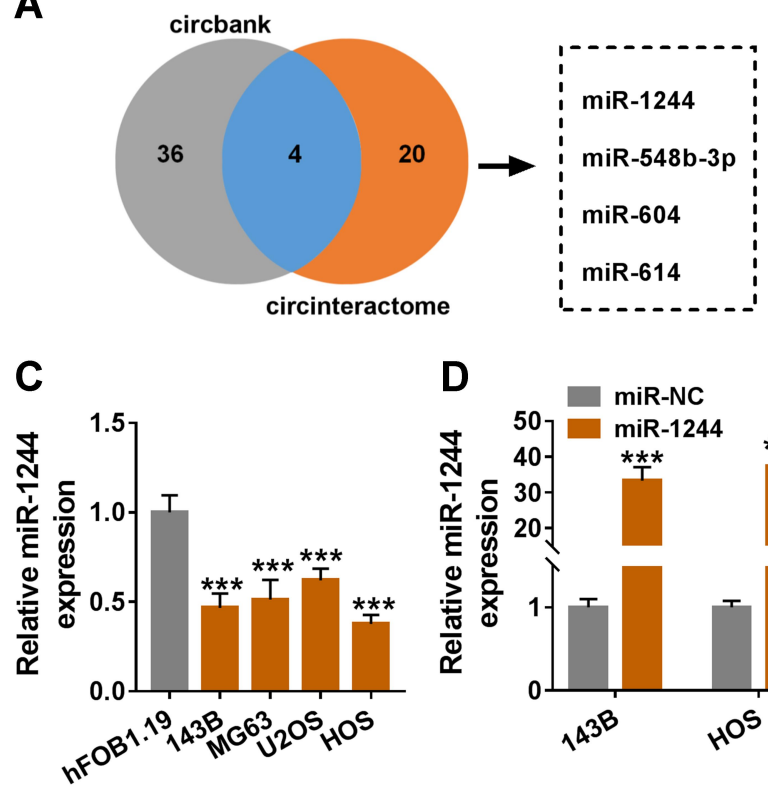

$\mathbf{F}$

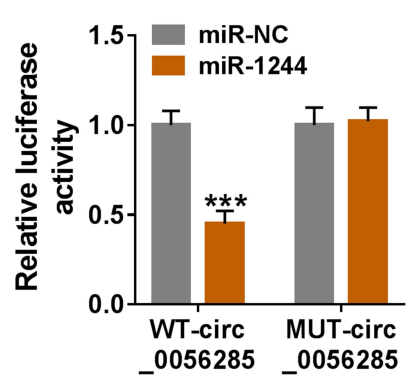

H

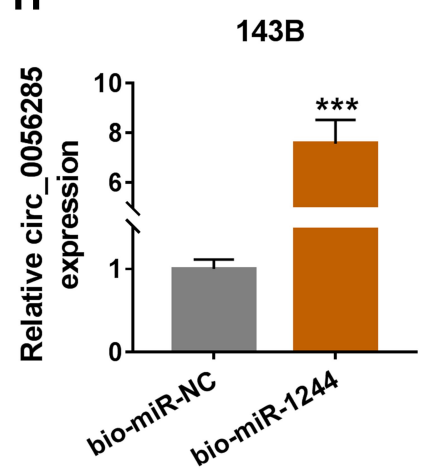

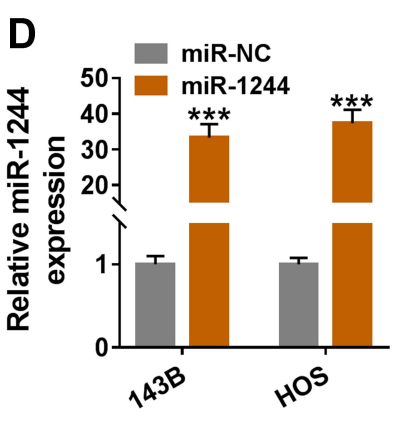

HOS

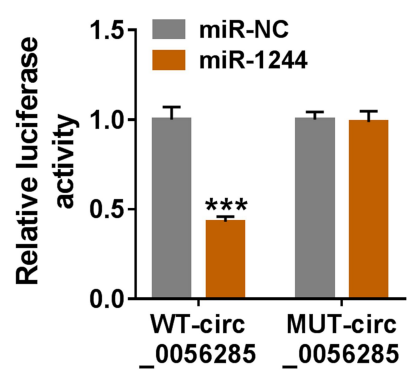

G

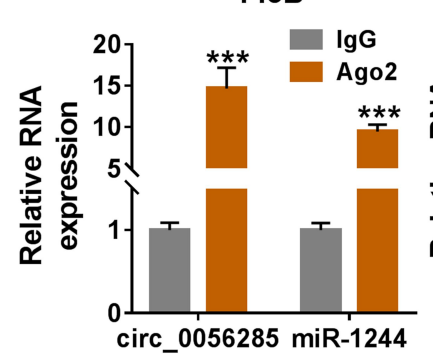

B

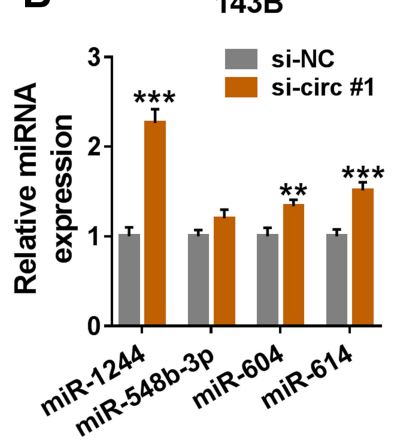

E

WT-circ_0056285 5' GCAGCCAUUCGAGAUAACUACUU 3' miR-1244 3' UUgGuagaguauguUugguUgaugaA 5'

MUT-circ_0056285 5' GCAGCCAUUCGAGAUUUGAUGAU 3'

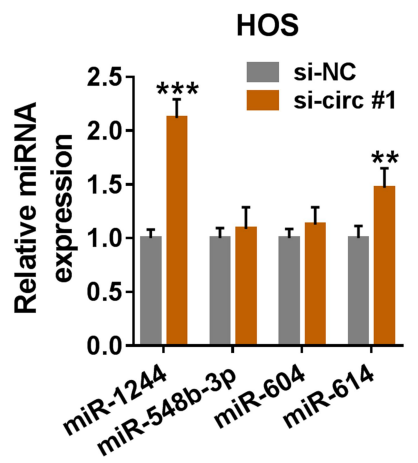

Figure 4 Circ_0056285 directly interacted with miR-1244. (A) Venn diagram showed miRNAs bound to circ_0056285 in Circbank and circinteractome databases. (B) The levels of four miRNAs (miR-I244, miR-548b-3p, miR-604 and miR-6I4) were tested in I43B and HOS cells transfected with si-NC or si-circ\#I. (C) MiR-I244 expression in hFOBI.19 and OS cell lines (143B, MG63, U2OS and HOS) was evaluated using qRT-PCR. (D) The expression of miR-I244 was tested in I43B and HOS cells transfected with miR-I244 or miR-NC. (E) The predicted binding sites of circ 0056285 and miR-1244 were exhibited. (F) I43B and HOS cells were co-transfected with WT-circ _ 0056285 or MUT-circ_0056285 and miR-I244 or miR-NC, and luciferase activity was detected by dual-luciferase reporter assay. (G and $\mathbf{H}$ ) The interaction between circ_0056285 and miR-I 244 was further verified using RIP assay and RNA pull-down assay. $* * P<0.01$, $* * * P<0.001$.

overexpression efficiency in 143B and HOS cells transfected with oe-circ_0056285 (***P<0.001, Figure 5D). To clarify the regulatory effect of circ_0056285 on TRIM44 expression, 143B and HOS cells were introduced with miR-NC, miR-1244, miR-1244+vector or
miR-1244+oe-circ_0056285. As shown in Figure 5E and $\mathrm{F}$, up-regulation of miR-1244 remarkably reduced TRIM44 protein level, while the impact was reversed after transfection with oe-circ_005628 $(* * * P<0.001)$. Moreover, circ_0056285 and TRIM44 were up-regulated, 
A

WT-TRIM44 3' UTR 5' GUGACCUGAGAGUCUAACUACUC 3' miR-1244 3' UUgGuagaguaUgUUUGGUUGAUGAA 5'

MUT-TRIM44 3' UTR 5' GUGACCUGAGAGUCUUUGAUGAC 3'
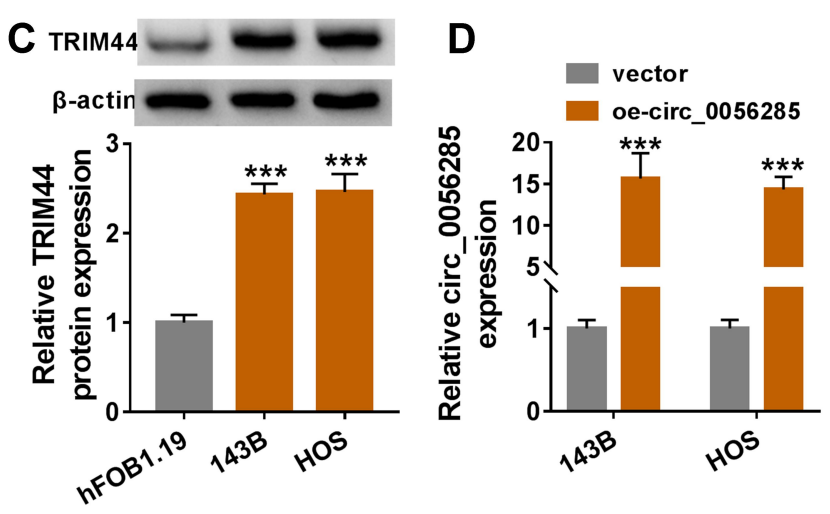

B

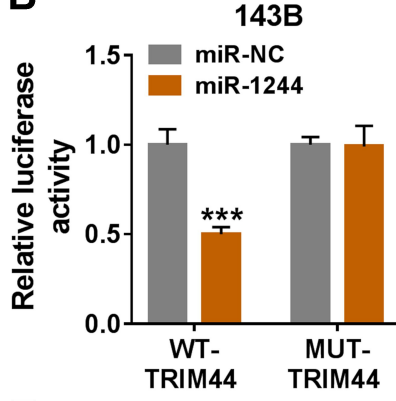

E
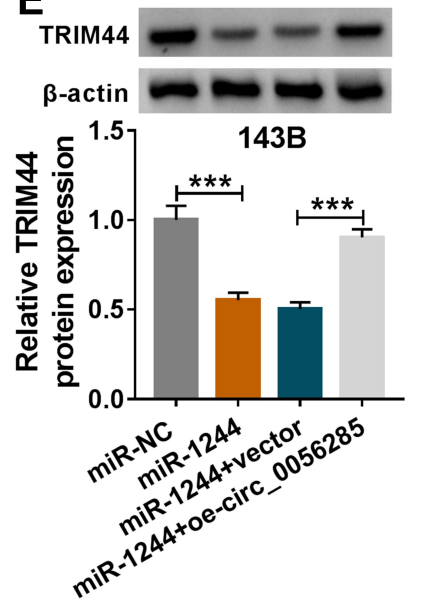

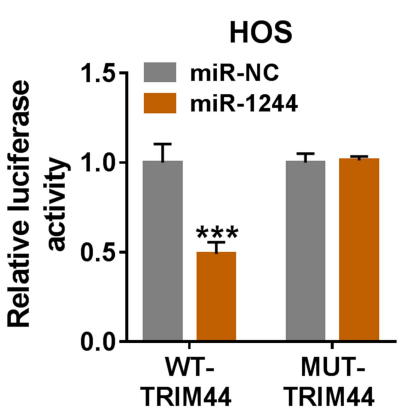

$\mathbf{F}$
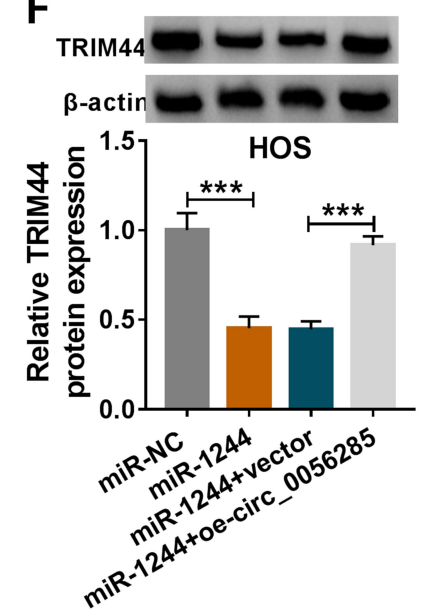

Figure 5 Circ_0056285 sponged miR-I244 to regulate TRIM44 expression. (A) The putative binding sites for miR-I244 and TRIM44 3'UTR were shown. (B) The relationship between miR-I244 and TRIM44 was validated by dual-luciferase reporter assay. (C) TRIM44 protein level was detected in hFOBI.19 and OS cell lines (I43B and HOS) using Western blot assay. (D) Circ_0056285 expression was measured in I43B and HOS cells transfected with vector or oe-circ_0056285. (E and F) I43B and HOS cells were introduced with miR-NC, miR-1244, miR-1244+vector or miR-1244+oe-circ_0056285, and TRIM44 protein expression was examined by Western blot assay. $* * * P<0.001$.

while miR-1244 was down-regulated in OS tissues $(\mathrm{n}=35)$ compared with normal tissues $(* * * P<0.001$, Figure S2A-C). Spearman correlation analysis suggested that miR-1244 was negatively correlated with circ_0056285 or TRIM44, while circ_0056285 was positively correlated with TRIM44 in OS tissues (Figure

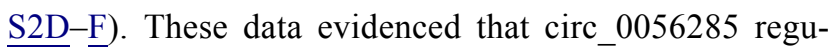
lated TRIM44 expression via sponging miR-1244.

\section{Inhibition of miR-1244 or}

\section{Overexpression of TRIM44 Reversed the Effect of Circ_0056285 Depletion on OS Progression}

Firstly, the inhibition efficiency of miR-1244 and the overexpression efficiency of TRIM44 were determined using qRT-PCR and Western blot $(* * * P<0.001$, Figure $6 \mathrm{~A}$ and B). Next, rescue experiments were performed in 143B and HOS cells transfected with si-NC, si-circ\#1, si-circ\#1+antiNC, si-circ\#1+anti-miR-1244, si-circ\#1+vector or si-circ\#1 +oe-TRIM44. CCK-8 assay and colony formation assay showed that miR-1244 down-regulation or TRIM44 upregulation partially abolished the reduction in cell proliferation induced by circ_0056285 silencing $(* * * P<0.001$, Figure 6C-E). In addition, knockdown of circ_0056285 accelerated OS cell apoptosis, while the effect was partly undermined after transfection with anti-miR-1244 or oeTRIM44 (***P<0.001, Figure 6F). Consistently, miR1244 down-regulation or TRIM44 up-regulation attenuated the activation of caspase 3 and caspase 9 caused by circ_0056285 knockdown $(* * * P<0.001$, Figure 6G and H). Moreover, depletion of circ_0056285 inhibited glycolysis by reducing glucose uptake, lactate product, ATP level and glycolysis-related protein (HK2 and LDHA) levels, whereas the effect was partly reversed after introduction with anti-miR-1244 or oe-TRIM44 $(* * * P<0.001, * * P=$ $0.004, * * P=0.002,{ }^{*} * P=0.002, * * P=0.008, * P=0.032$, $* * P=0.003$, or ${ }^{* *} P=0.002$, Figure $\left.6 \mathrm{I}-\mathrm{M}\right)$. These data suggested that miR-1244 knockdown or TRIM44 overexpression overturned the effect of circ_0056285 silencing on OS cell proliferation, apoptosis and glycolysis. 
A

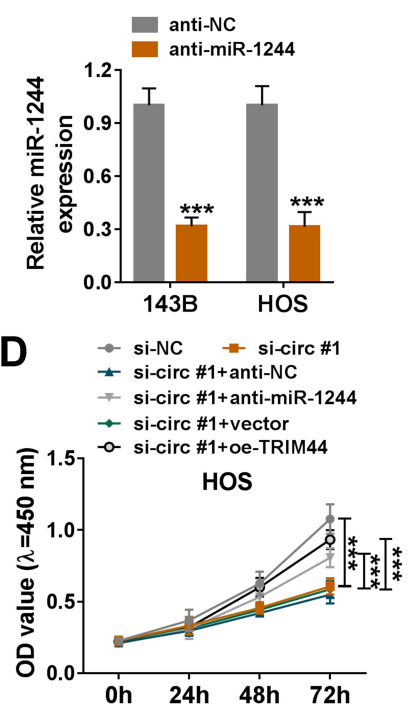

$\mathbf{F}$
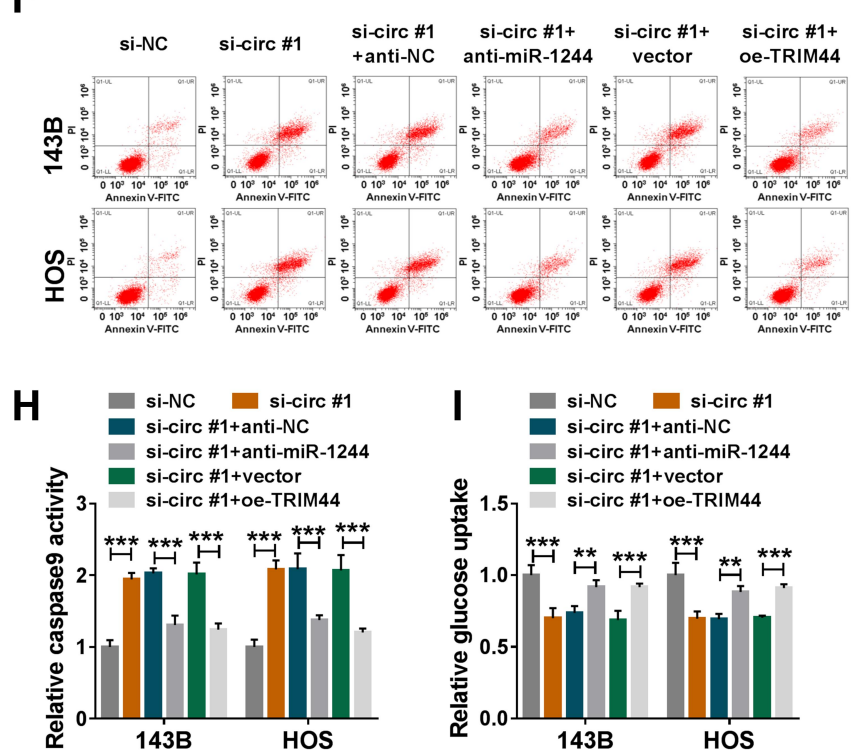

L

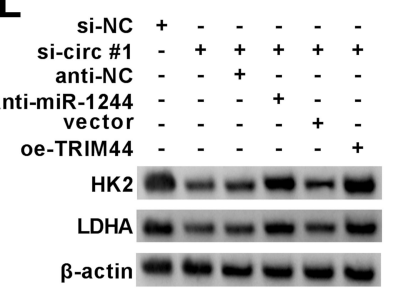

$143 B$
B
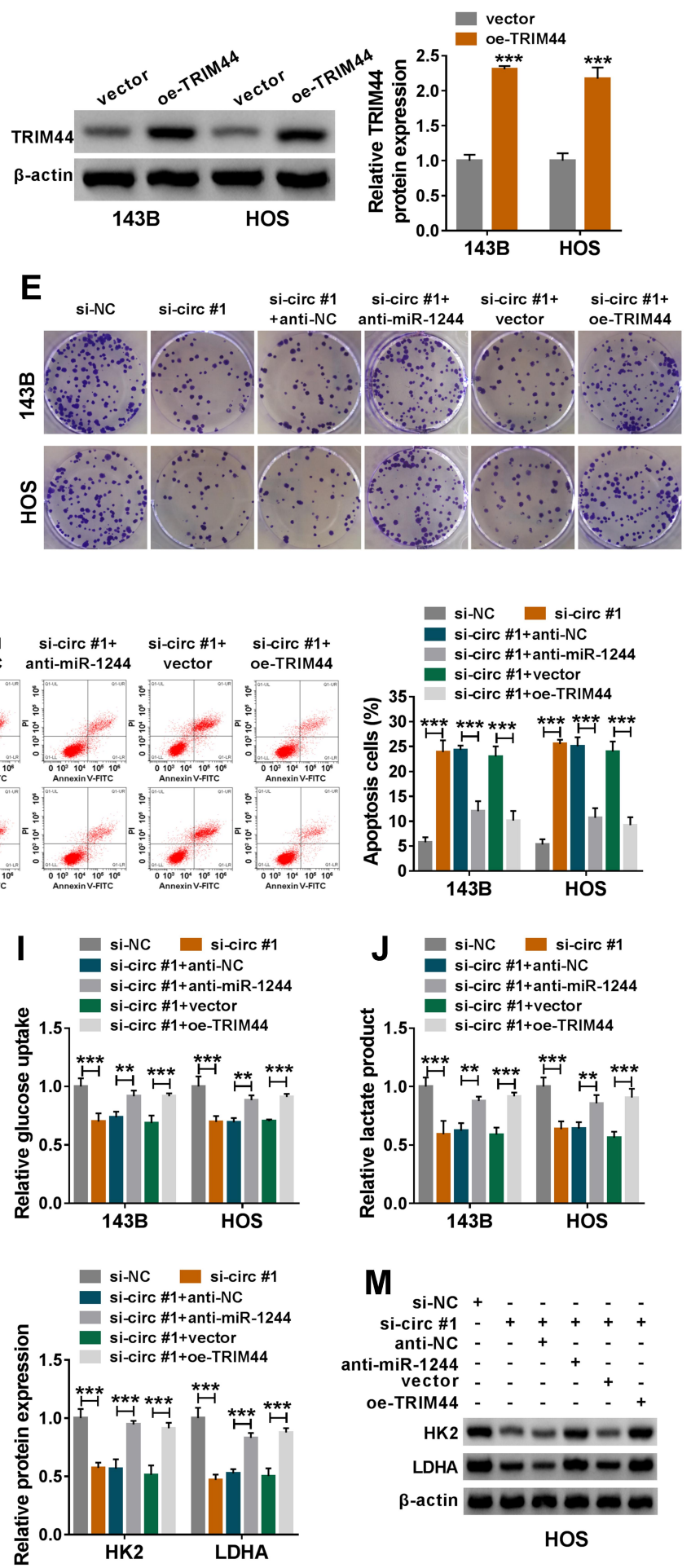

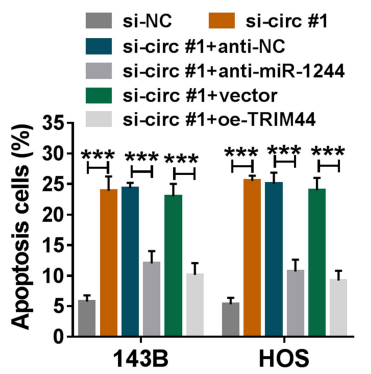

J si-NC si-circ \#1
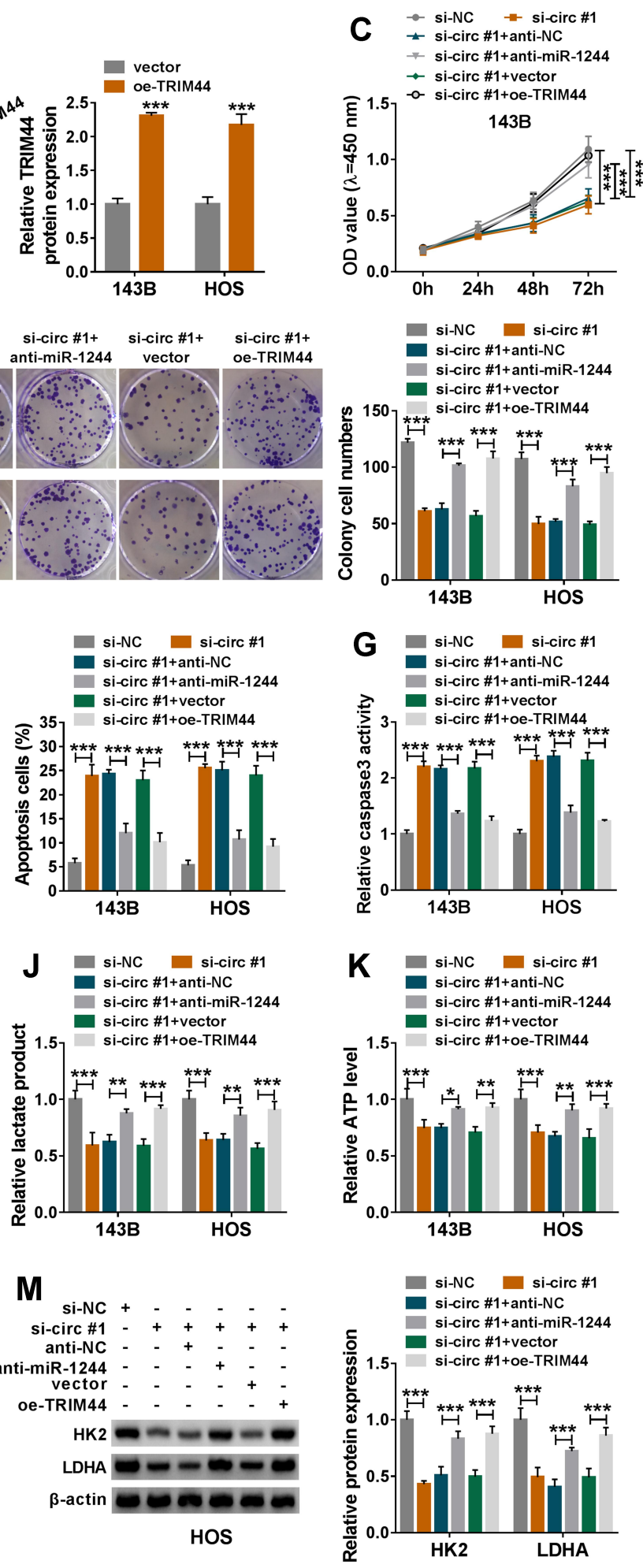

Figure 6 Inhibition of miR-I 244 or overexpression of TRIM44 reversed the effect of circ_0056285 depletion on OS progression. (A) The expression of miR-1244 was detected in 143B and HOS cells transfected with anti-NC or anti-miR-1244. (B) TRIM44 protein level was examined in I43B and HOS cells introduced with vector or oeTRIM44. (C-M) I43B and HOS cells were transfected with si-NC, si-circ\#I, si-circ\#I+anti-NC, si-circ\#I+anti-miR-I244, si-circ\#I+vector or si-circ\#I+oe-TRIM44, respectively. (C and D) Cell viability was assessed by CCK-8 assay. (E) The number of colonies was detected by colony formation assay. (F) Cell apoptosis was monitored by flow cytometry. ( $\mathbf{G}$ and $\mathbf{H}$ ) Caspase 3 and caspase 9 activities were tested by commercial kits. (I-K) After $48 \mathrm{~h}$ of transfection, glucose uptake, lactate product and ATP level were detected. ( $\mathbf{L}$ and $\mathbf{M})$ Western blot assay was utilized to detect the protein levels of HK2 and LDHA. $* P<0.05, * * P<0.0 \mathrm{I}, * * * P<0.00 \mathrm{I}$. 


\section{Circ_0056285 Knockdown Blocked} Tumor Growth in vivo

Further, to investigate the effect of circ_0056285 on tumorigenesis in vivo, 143B cells stably transfected with sh-NC or sh-circ were subcutaneously injected into the nude mice. Compared with sh-NC group, tumor volume and weight of sh-circ group were markedly reduced $(* * * P<0.001$, Figure $7 \mathrm{~A}$ and B). Knockdown of circ_0056285 resulted in a significant decrease in circ_0056285 expression and a marked increase in miR-1244 expression $(* * * P<0.001$, Figure $7 \mathrm{C}$ and D). Western blot analysis exhibited that the protein levels of TRIM44, HK2 and LDHA in sh-circ group were significantly lower than those in sh-NC group $(* * * P<$ 0.001 , Figure 7E). Besides, IHC assay showed that silencing of circ_0056285 markedly reduced Ki67-positive cells $\left({ }^{* *} P=0.008\right.$, Figure $\left.7 \mathrm{~F}\right)$. These data evidenced that circ_0056285 depletion impeded tumor growth in vivo.

\section{Circ_0056285 Was Highly Expressed in Serum Exosomes of OS Patients}

Next, we isolated exosomes from the serums of OS patients and healthy volunteers. The characteristics of these vesicles were observed with a TEM (Figure 8A). The particle size distribution was detected by NTA, and the results showed that the particle size range was $75-150 \mathrm{~nm}$ (Figure 8B). In addition, the presence of exosome markers (CD61, CD81 and TSG101) was confirmed by Western blot analysis (Figure 8C). Also, circ_0056285 and TRIM44 levels in serum exosomes of OS patients were significantly higher than those in healthy volunteers, while miR-1244 level had
A

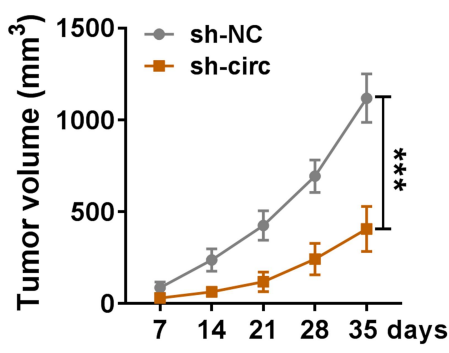

E

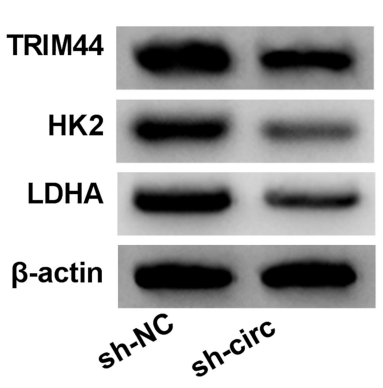

$\mathbf{F}$

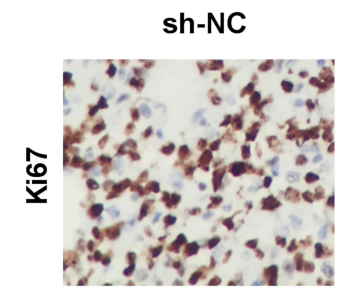

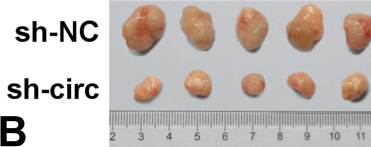

B
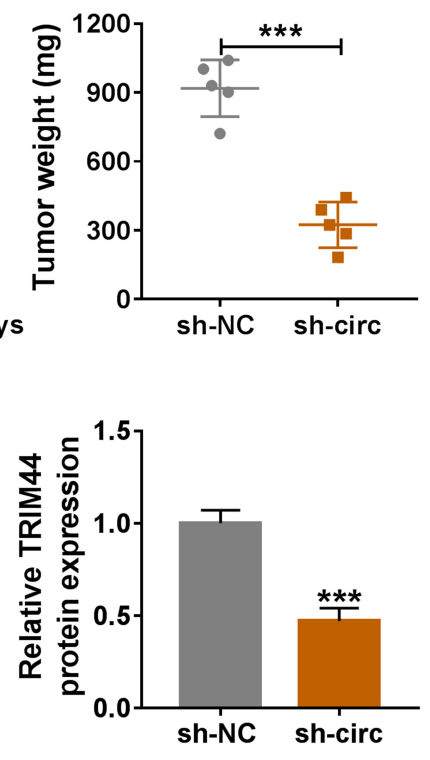
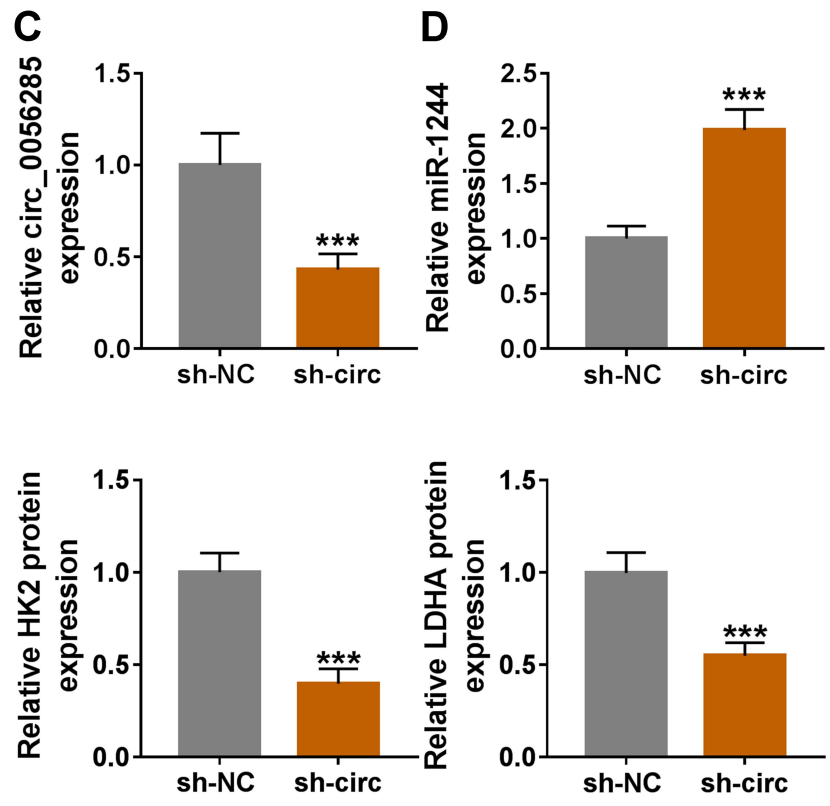

Figure 7 Circ_0056285 knockdown blocked tumor growth in vivo. I43B cells stably transfected with sh-NC or sh-circ were subcutaneously injected into the right-back of nude mice. (A) After 7 days, the growth curve of xenograft tumor was plotted. (B) After 35 days, the mice were sacrificed, and the tumors were weighed. (C and $\mathbf{D})$ The levels of circ_0056285 and miR-I 244 were measured using qRT-PCR. (E) The protein levels of TRIM44, HK2 and LDHA were detected by Western blot. (F) IHC assay was used to analyze Ki67 in xenograft tumor tissues. $* * P<0.01$, *** $P<0.001$. 
A

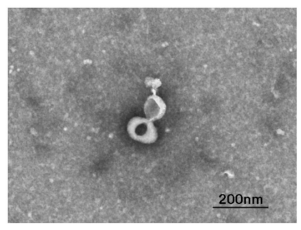

Normal

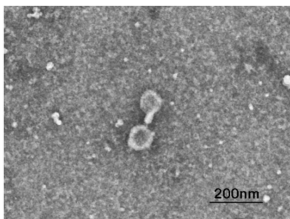

OS
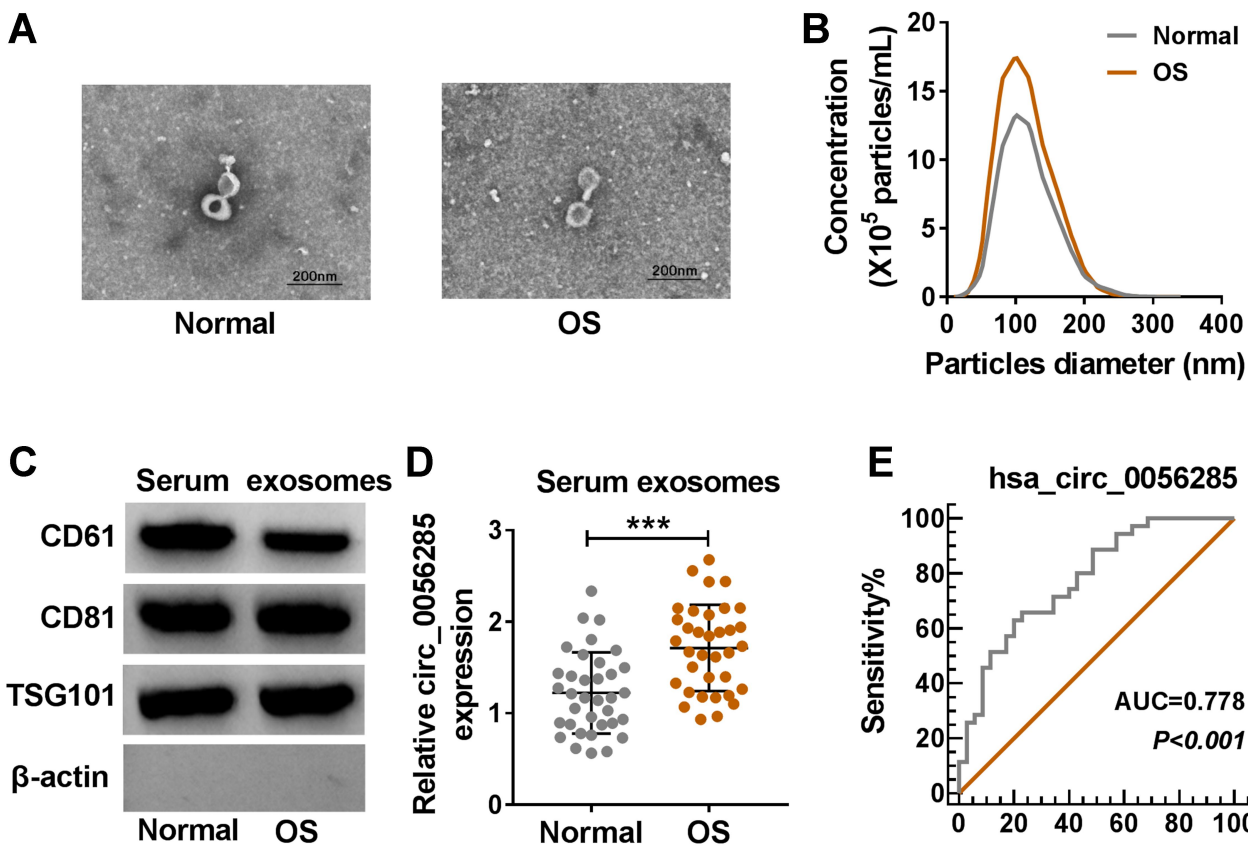

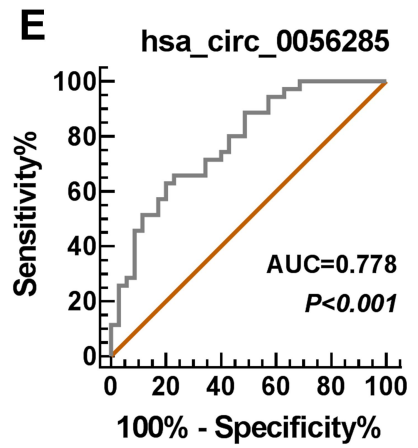

Figure 8 Circ_0056285 was highly expressed in serum exosomes of OS patients. (A) Representative images of serum exosomes were examined by TEM. (B) Size distribution was analyzed by NTA. (C) Exosome markers (CD6I, CD8I and TSGI0I) were detected by Western blot. (D) Circ_0056285 expression in serum exosomes of OS patients $(n=35)$ and healthy volunteers $(n=35)$ was measured by qRT-PCR. (E) ROC curve was used to evaluate the diagnostic value of exosomal hsa_circ_0056285 in OS. $* * * P<0.001$.

the opposite trend (Figures $8 \mathrm{D}$ and $\underline{\mathrm{S} 3 \mathrm{~A}}$ and $\underline{\mathrm{B}}$ ). Spearman correlation analysis showed that circ_0056285 in OS tissues was positively correlated with circ_0056285 in serum exosomes, while miR-1244 and TRIM44 in OS tissues were not associated with their in serum exosomes (Figure S3C-E). Furthermore, ROC curve showed that the level of exosomal hsa_circ_0056285 had high diagnostic value for OS (Figure 8E).

\section{Discussion}

With the development of high-throughput sequencing technology, abnormally expressed circRNAs have attracted widespread attention in tumor research. ${ }^{21}$ Numerous studies have revealed that circRNAs indirectly regulate gene expression by serving as competing endogenous RNAs (ceRNAs). ${ }^{22}$ Moreover, circRNAs regulate tumor development and progression by mediating a variety of biological processes, including cell proliferation, apoptosis, metastasis, and glycolysis. ${ }^{23}$ Among them, glycolysis is a hallmark of cancer, and hexokinase (HK) is the first step to catalyze glycolysis. ${ }^{24}$ Chen et al indicated that circRNA_100290 accelerated oral squamous cell carcinoma progression by promoting glycolysis through miR-378a/GLUT1 pathway. ${ }^{25}$ Cao et al disclosed that circRNF20 expedited tumor growth and glycolysis by functioning as a ceRNA for miR-487a and up-regulating HIF- $1 \alpha^{26}$ In the present research, circ_0056285 expression was overtly increased in osteosarcoma. Moreover, circ_0056285 was mainly expressed in cytoplasm, suggesting that circ_0056285 might be a sponge for miRNA. Functional experiments showed that circ_0056285 silencing inhibited OS cell progression by repressing cell proliferation and glycolysis and inducing apoptosis.

Bioinformatics software predicted four putative target miRNAs of circ_0056285, and miR-1244 was selected as a candidate for subsequent research. Previous studies have corroborated that miR-1244 acts as an inhibitor in various cancers. For example, Zhang et al revealed that miR-1244 ameliorated the malignancy of lung carcinoma via decreasing MEF2D expression. ${ }^{27}$ Additionally, miR-1244 impeded tumor progression and enhanced cisplatin sensitivity by modulating MEF2D. ${ }^{28}$ In ovarian cancer, knockdown of miR-1244 attenuated the inductive effect of LINC00504 on glycolysis. ${ }^{29}$ Yanbin et al suggested that miR-1244 expression was prominently decreased, and miR-1244 blocked cell proliferation in OS, ${ }^{15}$ which was consistent with our study. Furthermore, we first verified that circ_0056285 was a sponge for miR-1244.

Mounting evidence has unveiled that TRIM44 plays a vital role in various biological processes of tumors. 
Several investigations found that TRIM44 facilitated tumor occurrence and development through activation of AKT/mTOR signaling pathway. ${ }^{18,30,31}$ Furthermore, overexpressed TRIM44 in OS cells promoted OS progression by binding to miR-410. ${ }^{32}$ In the present study, we firstly verified the new ceRNA mechanism that circ_0056285 regulated TRIM44 expression by sponging miR-1244.

Exosomes are nanoscale extracellular vesicles that carry abundant functional biomolecules. ${ }^{33}$ Emerging evidence has demonstrated that tumor-derived exosomes participate in tumor microenvironment by transferring biomolecules to adjacent cells or distant organs. ${ }^{34}$ Additionally, exosomal circRNAs have emerged as potential therapeutic targets in a variety of tumors by promoting cell communication and tumor metastasis. ${ }^{35}$ In this research, we first discovered that circ_0056285 was markedly up-regulated in serum exosomes of OS patients, and exosomal circ_0056285 had high diagnostic value for OS.

In summary, these findings demonstrated that circ_0056285 sponged miR-1244 to elevate TRIM44 expression in OS. Importantly, circ_0056285 depletion suppressed OS cell progression and glycolysis via regulating miR-1244/TRIM44 axis. Therefore, this study might provide a new therapeutic target for OS.

\section{Disclosure}

The authors declare that they have no conflict of interest.

\section{References}

1. Whelan JS, Davis LE. Osteosarcoma, chondrosarcoma, and chordoma. J Clin Oncol. 2018;36(2):188-193. doi:10.1200/JCO.2017.75.1743

2. Luetke A, Meyers PA, Lewis I, Juergens H. Osteosarcoma treatmentwhere do we stand? A state of the art review. Cancer Treat Rev. 2014;40(4):523-532. doi:10.1016/j.ctrv.2013.11.006

3. Chen LL. The biogenesis and emerging roles of circular RNAs. Nat Rev Mol Cell Biol. 2016;17(4):205-211. doi:10.1038/nrm.2015.32

4. Shang Q, Yang Z, Jia R, Ge S. The novel roles of circRNAs in human cancer. Mol Cancer. 2019;18(1):6. doi:10.1186/s12943-018-0934-6

5. Wang C, Ren M, Zhao X, Wang A, Wang J. Emerging roles of circular RNAs in osteosarcoma. Med Sci Monit. 2018;24:7043-7050. doi:10.12659/MSM.912092

6. Ji X, Shan L, Shen P, He M. Circular RNA circ_001621 promotes osteosarcoma cells proliferation and migration by sponging miR-578 and regulating VEGF expression. Cell Death Dis. 2020;11(1):18. doi:10.1038/s41419-019-2204-y

7. Li S, Pei Y, Wang W, Liu F, Zheng K, Zhang X. Circular RNA 0001785 regulates the pathogenesis of osteosarcoma as a ceRNA by sponging miR-1200 to upregulate HOXB2. Cell Cycle. 2019;18 (11):1281-1291. doi:10.1080/15384101.2019.1618127

8. Jin Y, Li L, Zhu T, Liu G. Circular RNA circ_0102049 promotes cell progression as ceRNA to target MDM2 via sponging miR-1304-5p in osteosarcoma. Pathol Res Pract. 2019;215(12):152688. doi:10.1016/j. prp.2019.152688
9. Huang Q, Huang Q-Y, Sun Y, Wu S. High-throughput data reveals novel circular RNAs via competitive endogenous RNA networks associated with human intracranial aneurysms. Med Sci Monit. 2019;25:4819-4830. doi:10.12659/MSM.917081

10. Chen J, Liu G, Wu Y, et al. CircMYO10 promotes osteosarcoma progression by regulating miR-370-3p/RUVBL1 axis to enhance the transcriptional activity of beta-catenin/LEF1 complex via effects on chromatin remodeling. Mol Cancer. 2019;18(1):150. doi:10.1186/ s12943-019-1076-1

11. Palmini G, Marini F, Brandi ML. What is new in the miRNA world regarding osteosarcoma and chondrosarcoma? Molecules. 2017;22 (3):417. doi:10.3390/molecules22030417

12. Tian BG, Hua Z, Wang ZJ, Li J. Knockdown of microRNA-181a inhibits osteosarcoma cells growth and invasion through triggering NLRP3-dependent pyroptosis. Eur Rev Med Pharmacol Sci. 2020;24 (15):7922.

13. Wang Y, Yang J, Chen P, et al. MicroRNA-320a inhibits invasion and metastasis in osteosarcoma by targeting cytoplasmic polyadenylation element-binding protein 1. Cancer Med. 2020;9(8):2833-2845. doi:10.1002/cam4.2919

14. Zheng XM, Xu CW, Wang F. MiR-33b inhibits osteosarcoma cell proliferation through suppression of glycolysis by targeting Lactate Dehydrogenase A (LDHA). Cell Mol Biol (Noisy-Le-Grand). 2018;64(11):31-35. doi:10.14715/cmb/2018.64.11.6

15. Yanbin Z, Jing Z. CircSAMD4A accelerates cell proliferation of osteosarcoma by sponging miR-1244 and regulating MDM2 mRNA expression. Biochem Biophys Res Commun. 2019;516(1):102-111. doi:10.1016/j.bbrc.2019.05.182

16. Zheng J, Zhang Y, Zhi L, et al. The novel gene TRIM44L from orange-spotted grouper negatively regulates the interferon response. Fish Shellfish Immunol. 2019;92:746-755. doi:10.1016/j.fsi.2019.06.062

17. Yamada Y, Kimura N, Takayama KI, et al. TRIM44 promotes cell proliferation and migration by inhibiting FRK in renal cell carcinoma. Cancer Sci. 2020;111(3):881-890. doi:10.1111/cas.14295

18. Li CG, Hu H, Yang XJ, Huang CQ, Yu XQ. TRIM44 promotes colorectal cancer proliferation, migration, and invasion through the Akt/mTOR signaling pathway. Onco Targets Ther. 2019;12: 10693-10701. doi:10.2147/OTT.S228637

19. Kawabata H, Azuma K, Ikeda K, et al. TRIM44 is a poor prognostic factor for breast cancer patients as a modulator of NF-kappaB signaling. Int J Mol Sci. 2017;18(9):1931. doi:10.3390/ijms18091931

20. Liu S, Yin H, Ji H, Zhu J, Ma R. Overexpression of TRIM44 is an independent marker for predicting poor prognosis in epithelial ovarian cancer. Exp Ther Med. 2018;16(4):3034-3040. doi:10.3892/ etm.2018.6541

21. Shen B, Wang Z, Li Z, Song H, Ding X. Circular RNAs: an emerging landscape in tumor metastasis. Am J Cancer Res. 2019;9(4):630-643.

22. Zhu KP, Zhang CL, Ma XL, Hu JP, Cai T, Zhang L. Analyzing the interactions of mRNAs and ncRNAs to predict competing endogenous RNA networks in osteosarcoma chemo-resistance. Mol Ther. 2019;27(3):518-530. doi:10.1016/j.ymthe.2019.01.001

23. Zhong Y, Du Y, Yang X, et al. Circular RNAs function as ceRNAs to regulate and control human cancer progression. Mol Cancer. 2018;17 (1):79. doi:10.1186/s12943-018-0827-8

24. Xu S, Herschman HR, Tumor Agnostic A. Therapeutic strategy for hexokinase 1-null/hexokinase 2-positive cancers. Cancer Res. 2019;79(23):5907-5914. doi:10.1158/0008-5472.CAN-19-1789

25. Chen X, Yu J, Tian H, et al. Circle RNA hsa_circRNA_100290 serves as a ceRNA for miR-378a to regulate oral squamous cell carcinoma cells growth via Glucose transporter-1 (GLUT1) and glycolysis. J Cell Physiol. 2019;234(11):19130-19140. doi:10.1002/ jcp. 28692

26. Cao L, Wang M, Dong Y, et al. Circular RNA circRNF20 promotes breast cancer tumorigenesis and Warburg effect through miR-487a/ HIF-1alpha/HK2. Cell Death Dis. 2020;11(2):145. doi:10.1038/ s41419-020-2336-0 
27. Zhang R, Zhang Y, Li H. miR-1244/myocyte enhancer factor 2D regulatory loop contributes to the growth of lung carcinoma. DNA Cell Biol. 2015;34(11):692-700. doi:10.1089/dna.2015.2915

28. Li GJ, Zhao GQ, Yang JP, et al. Effect of miR-1244 on cisplatin-treated non-small cell lung cancer via MEF2D expression. Oncol Rep. 2017;37(6):3475-3483. doi:10.3892/or.2017.5624

29. Liu Y, He X, Chen Y, Cao D. Long non-coding RNA LINC00504 regulates the Warburg effect in ovarian cancer through inhibition of miR-1244. Mol Cell Biochem. 2020;464(1-2):39-50. doi:10.1007/ s11010-019-03647-z

30. Wei CY, Wang L, Zhu MX, et al. TRIM44 activates the AKT/mTOR signal pathway to induce melanoma progression by stabilizing TLR4. J Exp Clin Cancer Res. 2019;38(1):137. doi:10.1186/s13046-0191138-7

31. Xiong D, Jin C, Ye X, et al. TRIM44 promotes human esophageal cancer progression via the AKT/mTOR pathway. Cancer Sci. 2018;109(10):3080-3092. doi:10.1111/cas.13762
32. Wang H, Fang ZL, Zhang GH, Ma X. TRIM44, a crucial target of miR-410, functions as a potential oncogene in osteosarcoma. Onco Targets Ther. 2018;11:3637-3647. doi:10.2147/OTT.S163163

33. Sun D, Zhuang X, Zhang S, et al. Exosomes are endogenous nanoparticles that can deliver biological information between cells. $A d v$ Drug Deliv Rev. 2013;65(3):342-347. doi:10.1016/j.addr.2012.07. 002

34. Chen $\mathrm{R}, \mathrm{Xu} \mathrm{X}$, Qian Z, et al. The biological functions and clinical applications of exosomes in lung cancer. Cell Mol Life Sci. 2019;76 (23):4613-4633. doi:10.1007/s00018-019-03233-y

35. Shi X, Wang B, Feng X, Xu Y, Lu K, Sun M. circRNAs and exosomes: a mysterious frontier for human cancer. Mol Ther Nucleic Acids. 2020;19:384-392. doi:10.1016/j.omtn.2019.11.023

\section{Publish your work in this journal}

Cancer Management and Research is an international, peer-reviewed open access journal focusing on cancer research and the optimal use of preventative and integrated treatment interventions to achieve improved outcomes, enhanced survival and quality of life for the cancer patient.
The manuscript management system is completely online and includes a very quick and fair peer-review system, which is all easy to use. Visit http://www.dovepress.com/testimonials.php to read real quotes from published authors. 